\title{
$\begin{array}{ll}\text { Research Square } & \begin{array}{l}\text { Preprints are preliminary reports that have not undergone peer review. } \\ \text { They should not be considered conclusive, used to inform clinical practice, } \\ \text { or referenced by the media as validated information. }\end{array}\end{array}$
}

\section{The impact of psychosocial interventions on neonatal and maternal mortality in LMICs: a meta-analysis of randomised controlled trials}

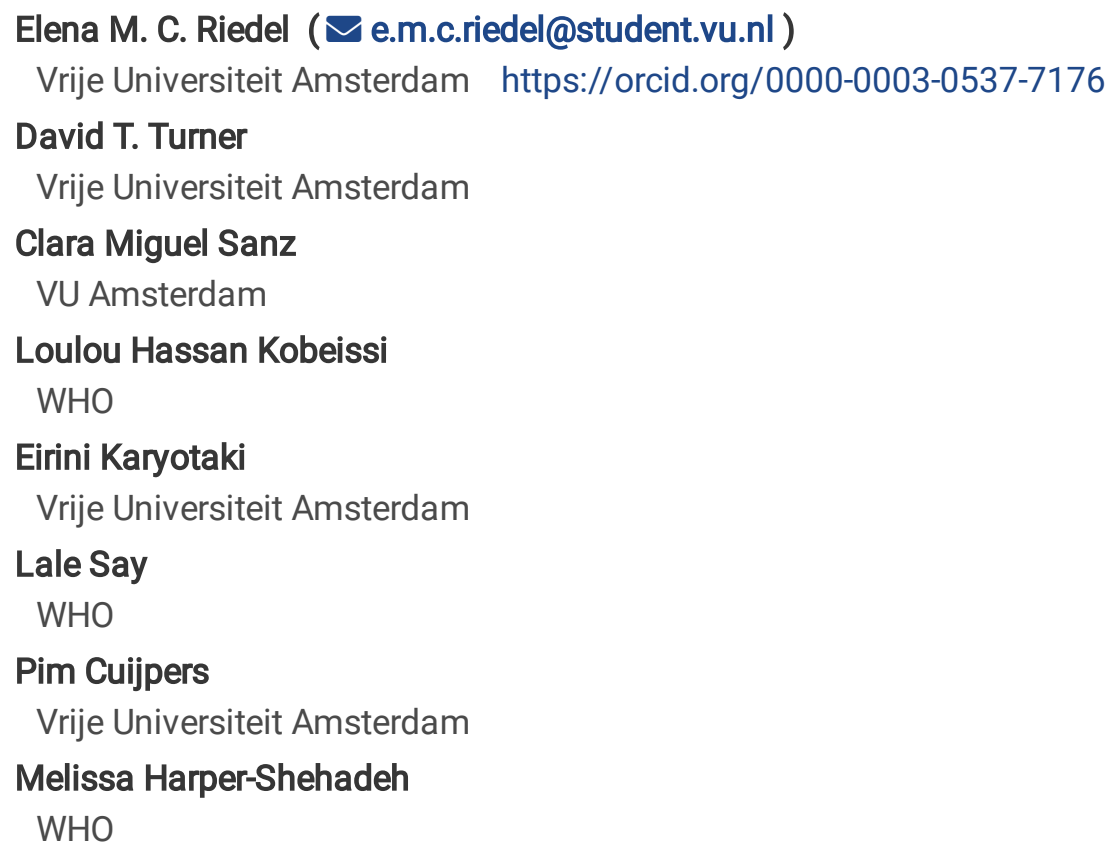

Keywords: Neonatal mortality, maternal mortality, psychosocial intervention, low- and middle-income countries, meta-analysis

Posted Date: January 6th, 2020

DOI: https://doi.org/10.21203/rs.2.20082/v1

License: (c) (i) This work is licensed under a Creative Commons Attribution 4.0 International License. Read Full License 


\section{Abstract}

\section{Background}

Despite progress in medical and skilled delivery care worldwide, neonatal and maternal mortality is still a major public health problem in resource-limited settings.

Objective

To determine whether psychosocial interventions (PSI) can reduce neonatal and maternal mortality in low-and middle-income countries, and which approaches are most promising.Methods Randomised controlled trials comparing a PSI with a control condition were identified through systematic searches in seven databases. Effects were pooled as risk ratios in randomeffects meta-analyses. Risk of bias was assessed using the Cochrane risk of bias tool, and publication bias was estimated. Sensitivity analyses were conducted to investigate sources of heterogeneity.

Results

Of 22 eligible RCTs (20 cluster randomised trials), the outcomes of 21 were synthesized for the outcome of neonatal mortality and 14 for maternal mortality. PSIs effectively reduced the risk of neonatal mortality by about $15 \%(\mathrm{RR} 0.85,95 \% \mathrm{Cl} 0.78-$ 0.94). The risk of maternal death was reduced by almost $21 \%$ ( RR $0.79,95 \% \mathrm{Cl} 0.68-0.93)$ with low levels of heterogeneity. There was low to medium risk of bias and no indication for substantial publication bias.

\section{Conclusions}

Results suggest that PSIs, mainly multi-method and group-based approaches, have the potential to substantially decrease the risk of maternal and neonatal death in low- and middle-income countries. The results of the latter outcome are marked by high heterogeneity and thus to be taken with caution.

\section{Introduction}

In the last decade, extensive efforts have been made to reduce deaths of mothers and newborns occurring during pregnancy, birth and the neonatal period (United Nations Foundation, 2018; WHO, 2014; Bhutta et al., 2014; Lawn et al., 2014). Despite widespread achievements, neonatal and maternal death tolls remain a major focus point on the global health agenda, as articulated in the United Nations Sustainable Development Goal 3 (UN, 2016; 2018a) and the Every Newborn WHO/UNICEF joint action plan (WHO, 2014), which is part of the UN Every Woman Every Child initiative (UN, 2018b). According to the latest WHO estimates, in 2018, the total number of newborn deaths worldwide was estimated at 2.5 million and the total worldwide maternal deaths was estimated in 2017 at 295,000. These rates cluster significantly among the most fragile and humanitarian settings.

Unsatisfying annual mortality reduction rates and substantial gaps in supply and quality of birth-related care remain to be a significant problem, especially in fragile as well as low- and middle-income countries (LMICs) (Lawn et al., 2014). The ambitious targets of $\leq 12$ neonatal deaths per 1,000 live births and less than 70 maternal deaths per 100,000 by the year 2030 (WHO, 2015) can only be reached if the striking inequity in health care is reduced, coupled with significant improvements in the quality, provision as well as access to basic medical care in line with global approve technical guidance before, during and after childbirth (Bhutta, 2014).

However, interventions that extend beyond the provision of medical care are needed to target high-risk newborn-care practices which are not only a matter of lacking care but of lacking knowledge or willingness for behavioural change. Improving individuals' knowledge and awareness of high-risk practices is necessary to empower them constructively and motivate behavioural change. The sociocultural context of women and newborns is directly associated with conducive health outcomes, as emphasized by the strategic objective 4 of the Every Newborn action plan (WHO, 2014). Psychosocial 
interventions are not focused on the provision of medical services, but rather target partners, families and communities of expectant mothers by providing psychological support, counselling or education and including those parties into intervention efforts.

The number of high-quality studies applying psychosocial interventions to reduce mortality has been increasing over the last years, while a statistical estimation of their impact remains warranted. We therefore present the meta-analytic results of psychosocial interventions in reducing the likelihood of neonatal and maternal mortality in low-resource settings.

\section{Methods}

\section{Protocol}

This paper is a sub-report within a broader project conducted in collaboration between the WHO Department of Reproductive Health and Research/Special Programme of Research, Development and Research Training in Human Reproduction (Geneva, $\mathrm{CH}$ ) and the Vrije Universiteit (Amsterdam, NL). The protocol is registered in the International Prospective Register of Systematic Reviews, no. CRD42018081410.

Search strategy

Systematic searches were conducted in seven databases up until December 2017 (Embase, PsyclNFO, PubMed, CINAHL, POPLINE, Global Health, and Global Index Medicus). Text and index variations of the keywords 'psychosocial intervention' as well as 'sexual and reproductive health' were combined. We restricted results to randomized controlled trials (RCT). An exemplary search string can be found in the Appendix.

\section{Selection of studies}

We included studies that were (1) randomised trials comparing a (2) psychosocial intervention to (3) a control condition with regard to (4) neonatal and/or maternal mortality and that were conducted in (5) a humanitarian setting or a LMIC as classified by The World Bank (2018).

Our definition of 'psychosocial' included interventions providing psychological and/or social services, such as counselling, education, or psychotherapy to individuals, groups or communities. It did not comprise interventions providing material resources nor interventions providing, medical, nutritional or pharmacological supplies only. Main components entailed by included interventions are described in Table 1. Eligible control conditions were no intervention, enhanced standard care, attention control or waitlist. Medical or pharmacological interventions were excluded as well as studies for which the full text was not retrievable or in a language other than English, French, Dutch, German, Greek, Spanish or Portuguese. Two authors (DT and ER) conducted the study selection using the online tool Covidence (www.covidence.org).

\section{Outcomes and data extraction}

Primary outcome was neonatal mortality (NNM), defined as the death of a liveborn child within the first 28 days of life. Further, we assessed maternal mortality, defined as death of a woman during or due to a cause related to pregnancy. Data were extracted by one author and cross-checked by another (ER, CM), solving discrepancies in discussion. Extracted data entailed information on setting, scope, type and delivery format of interventions and control conditions. For outcome data, the numbers of deaths at post-test were extracted alongside the corresponding total sample sizes of intervention and control groups. Following recommendations of the Cochrane Handbook for the meta-analysis of cluster trials (Higgins \& Green, 2011, ch. 16), we extracted direct estimates in the form of a Risk Ratio (RR) adjusted for clustering whenever available. Further, wherever available, intention-to-treat results were preferred over completers-only results. Authors were contacted in cases of missing or inconclusive outcome data.

Quality assessment 
We used five criteria of the Cochrane risk of bias assessment tool (Higgins \& Green, 2011, ch. 8) to examine risk of bias among included studies. These criteria were lack of random sequence generation and allocation concealment (selection bias), insufficient blinding of outcome assessors (detection bias), inappropriate handling of incomplete data (attrition bias) and any cluster-specific aspects not entailed by the other items. Following the Cochrane tool guidelines (ch. 16.3.2), these clusterspecific aspects were recruitment bias (e.g. due to differential recruitment among groups or recruitment happening after randomisation), baseline imbalance (e.g. due lack of stratification or matching of cluster pairs), attrition bias (due to loss of clusters) and incorrect statistical analysis.

Items were rated as 'risk present' when a risk was detected or could not be ruled out even after consultation of protocols and supplementary information. The overall risk of bias of a study was then determined from the sum of high-risk items. The assessment was done by three authors resolving conflicts in discussion (ER, CM, and DT).

Data synthesis

The effects of the intervention over control conditions were computed or extracted as RR for each study. When a study comprised several intervention groups which each met the inclusion criteria while being sufficiently distinct from one another, the effect for these groups was combined based on the recommendations for inclusion of multiple study groups of the Cochrane handbook (Higgins \& Green, 2011, ch. 16.5.4).

Meta-analyses were conducted only if there were at least five comparisons from eligible RCTs available (based on recommendations by Jackson \& Turner, 2017). Effects were pooled as RR, using a random effects model with $95 \%$ confidence intervals to account for the evident non-similarity between studies.

Statistical heterogeneity among included trials was examined by calculating the $\mathrm{I}^{2}$ statistic, which indicates the percentage of variability among studies that can be explained by heterogeneity rather than by random error/chance. Following the interpretation of Higgins et al. (2003), heterogeneity levels of around $25 \%$ were considered low, around 50\% moderate, and around $75 \%$ as high.

The potential influence of publication bias on the results was investigated by inspecting funnel plots created by CMA and Egger's test of the intercept. The extent of the influence was estimated by adjusting effect sizes with the trim-and-fill procedure (Duval \& Tweedie, 2000) and assessing the number of missing studies that would make effects insignificant (classic fail-safe N, Rosenthal, 1979).

Analyses were conducted using Comprehensive Meta-Analysis (CMA, vers. 3).

Sensitivity analyses

As pre-specified in the protocol, sensitivity analyses were conducted to investigate heterogeneity and the potential influence of risk of bias. Provided that there were at least five studies available, we investigated differential effects based on intervention type and level of risk of bias. Also, studies were consecutively removed to examine their individual influence on the overall effect.

\section{Results}

Characteristics of included studies

The flow chart of the study selection process is shown in Figure 1. The searches resulted in 6,478 records after removal of duplicates which were screened by title and abstract. Of these, 1,061 were retrieved and examined more closely, which led to the identification of 23 eligible RCTs. One more study had to be excluded because it reported only infant mortality (death within the first year of life). Of the 22 remaining studies, 21 were included in meta-analyses for the outcome of neonatal mortality and 14 for maternal mortality. 
Selected characteristics of the included studies are presented in Table 2. Except for two (Bashour et al., 2008; Villar et al., 1992), all studies were cluster-RCTs. The average and median sample sizes were 18,833 and 12,784 , respectively. Only four studies included fewer than 5,000 participants, and only one fewer than 1,000. 14 studies had been conducted in western (Syria), south (Pakistan, India, Nepal, Bangladesh) and southeast Asia (Vietnam), six studies in Africa (Guinea Bissau, Malawi, Tanzania, Ghana, South Africa), and two studies in multiple locations.

Details on the type and delivery format of included interventions are described in Table 2. More thorough explanations of the components are provided in Table 1.

Table 1. Components and formats of included interventions.

Component Description

Counselling, Comprising (among others) promotion of good prenatal care practices, birth preparedness and education \& facility-based delivery; teaching registration of danger signs and adequate emergency behaviour; information uncovering of false traditional beliefs about unsafe birth practices; employing means of visualisation adapted to the cultural context such as performance, folklore, video documentary.

Support Providing emotional as well as practical support before, during and after giving birth; facilitating referral to professional medical care; strengthening social support and involving family members; health monitoring.

Training Training of community health workers or existing traditional birth attendants in counselling and promotion strategies and communication skills; training of pregnant women in emergency behaviours.

Participatory Facilitation of regular collective meetings in the form of 'health groups' or 'women's groups'; approaches facilitation of communication and collaboration between different stakeholders (medical centre \&

facilitation staff, community heads/opinion leaders, volunteer health workers, women's unions, families and women); facilitation of participatory action cycles to identify perinatal problems and strategies to address them on a local scale.

\begin{tabular}{ll}
\hline Format & \\
$\begin{array}{l}\text { Individual/ } \\
\text { home } \\
\text { visits }\end{array}$ & $\begin{array}{c}\text { Community/social health workers, midwives or trained volunteers visiting pregnant women in their } \\
\text { homes/communities, usually delivering individual counselling or education, examination and } \\
\text { facilitation of referral etc. }\left(8^{*}\right)\end{array}$ \\
$\begin{array}{c}\text { Interventions consisting of (facilitation of) group sessions; participatory learning and action cycles } \\
\text { involving different community members; usually delivered by project staff, community workers, } \\
\text { or trained peers. }\left(8^{*}\right)\end{array}$ \\
$\begin{array}{c}\text { Multi- } \\
\text { method/ } \\
\text { 'package' }\end{array}$ & \begin{tabular}{c} 
usually large-scale intervention. $\left(6^{*}\right)$ \\
\hline
\end{tabular} \\
\hline
\end{tabular}

* Number of studies delivered in described format.

Table 2. Characteristics of selected studies.

Note. $N_{\text {Int }} / N_{\text {Ctrl }}$ : Sample size of intervention/control group; RoB: Risk of Bias index based on five items of the Cochrane tool; LHW: Lady (community) health worker; VHW: Voluntary health worker; SC: standard care; eSC: enhanced standard care (e.g., additional strengthening of health facilities in both groups alike).

a Outcome for which study has been included in a meta-analysis. NND: Neonatal death; SB: Stillbirth; MD: Maternal death.

${ }^{\mathrm{b}} \mathrm{N}$ refers to participants assessed and analysed for outcomes of interest; total study sample sizes may be larger.

${ }^{\mathrm{C}}$ Risk of Bias. Maximum score (highest possible risk) $=4$ for individual trials and $=5$ for cluster RCTs. Indicated index $=$ study score/maximum score*100 (<33.34 = low; 33.34-66.66 = medium; >66.66 = high RoB).

* Individual trial (all others are cluster RCT 


\begin{tabular}{|c|c|c|c|c|c|c|c|c|}
\hline Study & Country & Outcome $^{\mathrm{a}}$ & Intervention & $N_{\text {INT }}{ }^{b}$ & Ctrl & $N_{\text {CTRL }}$ & Post-test & RoB \\
\hline $\begin{array}{l}\text { Baqui et al., } \\
2008\end{array}$ & Bangladesh & NND, SB & $\begin{array}{l}\text { Individual (home visits) } \\
\text { \& groups; } \\
\text { counselling/education, } \\
\text { promotion of good } \\
\text { care practices and } \\
\text { birth preparedness, } \\
\text { referral. }\end{array}$ & 18,556 & $\mathrm{SC}$ & 19,497 & $\begin{array}{l}2.5 \\
\text { years }\end{array}$ & low \\
\hline $\begin{array}{c}* \text { Bashour et } \\
\text { al., } 2008\end{array}$ & Syria & NND & $\begin{array}{l}\text { Individual (home visits); } \\
\text { counselling/education, } \\
\text { examination, support. }\end{array}$ & 284 & $\mathrm{SC}$ & 296 & 4 months & high \\
\hline $\begin{array}{l}\text { Bhutta et al., } \\
2008\end{array}$ & Pakistan & $\begin{array}{l}\text { NND, SB, } \\
\text { MD }\end{array}$ & $\begin{array}{l}\text { Multi-method; village } \\
\text { health committees, } \\
\text { home visits, training } \\
\text { of LHWs and } \\
\text { traditional birth } \\
\text { attendants in } \\
\text { counselling and } \\
\text { promotion strategies, } \\
\text { group education } \\
\text { sessions. }\end{array}$ & 3,064 & $\mathrm{SC}$ & 2,778 & 2 years & high \\
\hline $\begin{array}{l}\text { Bhutta et al., } \\
2011\end{array}$ & Pakistan & $\begin{array}{l}\text { NND, SB, } \\
\text { MD }\end{array}$ & $\begin{array}{l}\text { Multi-method; village } \\
\text { health committees, } \\
\text { home visits, training } \\
\text { of LHWs and } \\
\text { traditional birth } \\
\text { attendants in } \\
\text { counselling and } \\
\text { promotion strategies, } \\
\text { group educ. sessions, } \\
\text { video docu drama for } \\
\text { pregnancy and } \\
\text { newborn care. }\end{array}$ & 12,391 & $\mathrm{SC}$ & 11,443 & 2 years & low \\
\hline $\begin{array}{l}\text { Boone et al., } \\
2016\end{array}$ & $\begin{array}{l}\text { Guinea } \\
\text { Bissau }\end{array}$ & NND, MD & $\begin{array}{l}\text { Multi-method; groups, } \\
\text { village health clubs, } \\
\text { visualisation, home } \\
\text { visits, counselling. }\end{array}$ & 6,729 & $\mathrm{SC}$ & 6,894 & $\begin{array}{l}2.5 \\
\text { years }\end{array}$ & medium \\
\hline $\begin{array}{l}\text { Boone et al., } \\
2017\end{array}$ & India & NND, MD & $\begin{array}{l}\text { Multi-method; health } \\
\text { education using } \\
\text { performance/folklore, } \\
\text { participatory } \\
\text { discussion groups, } \\
\text { home visits. }\end{array}$ & 6,400 & $\mathrm{SC}$ & 5,545 & 3 years & medium \\
\hline $\begin{array}{l}\text { Colbourn et } \\
\text { al., } 2013\end{array}$ & Malawi & $\begin{array}{l}\text { NND, SB, } \\
\text { MD }\end{array}$ & $\begin{array}{l}\text { Group; facilitation of } \\
\text { participatory action- } \\
\text { learning cycle to } \\
\text { identify and address } \\
\text { perinatal problems. }\end{array}$ & 5,080 & $\mathrm{SC}$ & 4,912 & 2 years & low \\
\hline $\begin{array}{l}\text { Darmstadt et } \\
\text { al., } 2010\end{array}$ & Bangladesh & NND, SB & $\begin{array}{l}\text { Individual (home visits); } \\
\text { education, health } \\
\text { monitoring. }\end{array}$ & 4,729 & $\mathrm{SC}$ & 5,350 & 3 years & medium \\
\hline $\begin{array}{l}\text { Fottrell et } \\
\text { al., } 2013\end{array}$ & Bangladesh & $\begin{array}{l}\text { NND, SB, } \\
\text { MD }\end{array}$ & $\begin{array}{l}\text { Group; education, } \\
\text { facilitation of } \\
\text { participatory action- } \\
\text { learning cycle to } \\
\text { identify and address } \\
\text { perinatal problems. }\end{array}$ & 9,106 & eSC & 8,834 & 2 years & low \\
\hline $\begin{array}{l}\text { Hanson et } \\
\text { al., } 2015\end{array}$ & Tanzania & NND & $\begin{array}{l}\text { Individual (home visits); } \\
\text { counselling, } \\
\text { promotion of facility- } \\
\text { based delivery. }\end{array}$ & 24,381 & $\mathrm{SC}$ & 23,307 & 6 years & low \\
\hline $\begin{array}{l}\text { Kirkwood et } \\
\text { al., } 2013\end{array}$ & Ghana & NND & $\begin{array}{l}\text { Individual (home visits); } \\
\text { counselling, } \\
\text { promotion of good } \\
\text { care practices. }\end{array}$ & 8,035 & $\mathrm{SC}$ & 8,294 & 1 year & low \\
\hline
\end{tabular}




\begin{tabular}{|c|c|c|c|c|c|c|c|c|}
\hline $\begin{array}{l}\text { Kumar et al., } \\
2008\end{array}$ & India & NND, SB & $\begin{array}{l}\text { Multi-method; individual } \\
\text { (home visits) \& } \\
\text { communal meetings; } \\
\text { counselling/education } \\
\text { on good care } \\
\text { practices. }\end{array}$ & 1,581 & $\mathrm{SC}$ & 1,143 & 1 year & medium \\
\hline $\begin{array}{r}\text { Lewycka et } \\
\text { al., } 2013\end{array}$ & Malawi & NND, MD & $\begin{array}{l}\text { Group; education, } \\
\text { facilitation of } \\
\text { participatory action- } \\
\text { learning cycle to } \\
\text { identify and address } \\
\text { perinatal problems. }\end{array}$ & 14,064 & $\mathrm{SC}$ & 5,059 & 3 years & low \\
\hline
\end{tabular}

\begin{tabular}{|c|c|c|c|c|c|c|c|c|}
\hline $\begin{array}{l}\text { Manandhar et } \\
\text { al., } 2004\end{array}$ & Nepal & $\begin{array}{r}\text { NND, } \\
\text { SB, } \\
\text { MD }\end{array}$ & $\begin{array}{l}\text { Group; education, } \\
\text { facilitation of } \\
\text { participatory action- } \\
\text { learning cycle to identify } \\
\text { and address perinatal } \\
\text { problems. }\end{array}$ & 2,972 & $\mathrm{eSC}$ & 3,303 & 2 years & medium \\
\hline $\begin{array}{l}\text { More et al., } \\
2012\end{array}$ & India & $\begin{array}{r}\text { NND, } \\
\text { SB, } \\
\text { MD }\end{array}$ & $\begin{array}{l}\text { Group; education, } \\
\text { facilitation of } \\
\text { participatory action- } \\
\text { learning cycle to identify } \\
\text { and address perinatal } \\
\text { problems. }\end{array}$ & 9,155 & SC & 9,042 & 3 years & medium \\
\hline $\begin{array}{l}\text { Nair et al., } \\
\quad 2017\end{array}$ & India & $\begin{array}{l}\text { NND, } \\
\text { SB }\end{array}$ & $\begin{array}{l}\text { Individual (home visits) \& } \\
\text { groups; } \\
\text { counselling/education, } \\
\text { promotion of good care } \\
\text { practices. }\end{array}$ & 2,814 & eSC & 2,967 & $\begin{array}{l}1.5 \\
\text { years }\end{array}$ & low \\
\hline $\begin{array}{l}\text { Pasha et al., } \\
2013\end{array}$ & $\begin{array}{l}\text { Ind, } \\
\text { Pak, } \\
\text { Ken, } \\
\text { Zmb, } \\
\text { Gtm, } \\
\text { Arg }\end{array}$ & $\begin{array}{r}\text { NND, } \\
\text { SB, } \\
\text { MD }\end{array}$ & $\begin{array}{l}\text { Multi-method; community } \\
\text { mobilisation through } \\
\text { village groups, training, } \\
\text { education of families. }\end{array}$ & 56,223 & $\mathrm{SC}$ & 55,306 & 3 years & medium \\
\hline $\begin{array}{l}\text { Persson et al., } \\
\quad 2013\end{array}$ & Vietnam & $\begin{array}{l}\text { NND, } \\
\text { SB, } \\
\text { MD }\end{array}$ & $\begin{array}{l}\text { Group; facilitation of health } \\
\text { groups including medical } \\
\text { staff, VHWs, community } \\
\text { and population } \\
\text { representatives and } \\
\text { women's union } \\
\text { representatives. }\end{array}$ & 11,906 & $\mathrm{SC}$ & 10,655 & 3 years & low \\
\hline $\begin{array}{l}\text { Tomlinson et } \\
\text { al., } 2014\end{array}$ & $\begin{array}{l}\text { South } \\
\text { Africa }\end{array}$ & $\begin{array}{r}\text { NND, } \\
\text { SB, } \\
\text { MD }\end{array}$ & $\begin{array}{l}\text { Individual (home visits); } \\
\text { education \& information. }\end{array}$ & 1,798 & eSC & 2,119 & $\begin{array}{l}12 \\
\text { weeks }\end{array}$ & low \\
\hline $\begin{array}{r}\text { Tripathy et } \\
\text { al., } 2010\end{array}$ & India & $\begin{array}{r}\text { NND, } \\
\text { SB, } \\
\text { MD }\end{array}$ & $\begin{array}{l}\text { Group; facilitation of } \\
\text { participatory action- } \\
\text { learning cycle to identify } \\
\text { and address perinatal } \\
\text { problems. }\end{array}$ & 9,770 & eSC & 9,260 & 3 years & low \\
\hline $\begin{array}{l}\text { Tripathy et } \\
\text { al., } 2016\end{array}$ & India & $\begin{array}{r}\text { NND, } \\
\text { SB, } \\
\text { MD }\end{array}$ & $\begin{array}{l}\text { Group; facilitation of } \\
\text { participatory action- } \\
\text { learning cycle to identify } \\
\text { and address perinatal } \\
\text { problems. }\end{array}$ & 3,700 & eSC & 3,519 & 2 years & low \\
\hline $\begin{array}{l}\text { *Villar et al., } \\
1992\end{array}$ & $\begin{array}{l}\text { Arg, } \\
\text { Bra, } \\
\text { Cub, } \\
\text { Mex }\end{array}$ & SB & $\begin{array}{l}\text { Individual (home visits); } \\
\text { counselling \& } \\
\text { psychosocial support } \\
\text { (strengthening social } \\
\text { network). }\end{array}$ & 1,033 & $\mathrm{SC}$ & 1,040 & $\begin{array}{l}1.5 \\
\text { months }\end{array}$ & low \\
\hline
\end{tabular}

The most frequent components entailed in interventions were a) individual home visits to new or expectant mothers, providing counselling, education and psychosocial support (13 studies) and b) approaches to identify and address perinatal challenges on a community level, such as the facilitation of participatory action-learning cycles through group meetings and community 
mobilisation (14 studies). Six studies were multi-method 'packages' including several components in a comprehensive intervention. Most control conditions consisted of no intervention/standard care, six comprised enhanced standard care in the form of strengthening of health services or a minimal version of the intervention.

All interventions relied on a task-sharing approach, that is, shifting of tasks and responsibilities to health workers that need not necessarily be highly educated, so as to increase coverage and cost-effectiveness. Most interventions were thus delivered by community or social health workers (CHW or SHW), some by midwives or volunteers recruited from the communities. Supporters were usually trained and supervised regularly by the research team or other involved professional staff. Some interventions also involved local 'traditional birth attendants' (e.g. Bhutta et al., 2008 \& 2011; Darmstadt et al., 2010) by providing training to them or strengthening collaboration between them and trained CHWs.

Risk of bias

Overall, the risk of bias in the current sample of studies was modest (Table 2). It varied among study settings and intervention types, with the majority of studies (13 of 22) rated as low risk, 7 of 22 as medium risk and only two studies rated as high risk. Of these latter, one was an individual trial (Bashour et al., 2008) and one was an implementation trial (Bhutta et al., 2008) which was not as rigorously designed and powered as its upscaled version which was rated as low risk (Bhutta et al., 2011).

Neonatal mortality

The rate of neonatal deaths was significantly lower in groups having received psychosocial interventions compared to control groups (RR $0.85,95 \% \mathrm{Cl} 0.78-0.94, p<.01$; Forest plot in Figure A-1). This effect suggests that the risk for new-borns to die within the first 28 days was about 15\% lower in intervention compared to control groups among the 21 included studies. Statistical heterogeneity among studies was high $(276,95 \% \mathrm{Cl} 64-84)$. Removal of four outliers reduced heterogeneity to a moderate extent $\left(R^{2} 40,95 \% \mathrm{Cl} 0-65\right)$ while the overall effect increased to $18 \%$ (RR 0.82, 95\% $\left.\mathrm{Cl} 0.76-0.88, p<.001\right)$. An overview of all results including further specification for early and late neonatal mortality is provided in Table 3.

Table 3. Overview of results of random effects meta-analyses and sensitivity analyses.

\begin{tabular}{|c|c|c|c|c|c|c|}
\hline \multirow[b]{2}{*}{ Analysis } & \multicolumn{3}{|c|}{ Effect size } & \multicolumn{3}{|c|}{ Heterogeneity } \\
\hline & $\mathrm{N}$ & Risk Ratio & $95 \% \mathrm{CI}$ & $z$-value & $I^{2}$ & $95 \% \mathrm{CI}$ \\
\hline Neonatal Mortality & 21 & $0.85^{* *}$ & $0.78 ; 0.94$ & -3.34 & $76.91^{* * *}$ & $64 ; 84$ \\
\hline \multicolumn{7}{|l|}{ Risk of bias } \\
\hline High RoB excluded ${ }^{\mathrm{a}}$ & 19 & $0.86^{* *}$ & $0.78 ; 0.95$ & -3.10 & $77.45^{* * *}$ & $64 ; 84$ \\
\hline Only low RoB (high/med. excl.) ${ }^{b}$ & 12 & $0.84 * * *$ & $0.76 ; 0.92$ & -3.49 & $66.98^{* * *}$ & $29 ; 81$ \\
\hline \multicolumn{7}{|l|}{ Outliers } \\
\hline Outliers excluded $^{\mathrm{C}}$ & 17 & $0.82 * * *$ & $0.76 ; 0.88$ & -5.41 & $40.38 *$ & $0 ; 65$ \\
\hline Only overestimating outlier excl. ${ }^{\mathrm{d}}$ & 20 & $0.87^{* *}$ & $0.80 ; 0.95$ & -2.99 & $73.91 * * *$ & $57 ; 82$ \\
\hline Only underest. outliers excl. ${ }^{\mathrm{e}}$ & 18 & $0.80 * * *$ & $0.74 ; 0.87$ & -5.33 & $52.91 * * *$ & $7 ; 71$ \\
\hline \multicolumn{7}{|l|}{ Intervention type } \\
\hline Individual approaches & 7 & 0.93 & $0.81 ; 1.06$ & -1.08 & 44.05 & $0 ; 75$ \\
\hline Group approaches & 8 & $0.85^{\natural}$ & $0.72 ; 1.01$ & -1.83 & $84.01 * * *$ & $68 ; 90$ \\
\hline Multi-method approaches & 6 & $0.82 *$ & $0.68 ; 0.99$ & -2.11 & $86.46^{* * *}$ & $70 ; 92$ \\
\hline $\begin{array}{l}\text { Maternal Mortality } \\
\text { Risk of bias }\end{array}$ & \multicolumn{5}{|c|}{ Risk of bias } & $0 ; 51$ \\
\hline High RoB excluded ${ }^{f}$ & 13 & $0.79^{* *}$ & $0.66 ; 0.94$ & -2.65 & 12.08 & $0 ; 55$ \\
\hline \multicolumn{7}{|l|}{ Intervention type } \\
\hline Group approaches & 8 & $0.73^{* *}$ & $0.58 ; 0.91$ & -2.76 & 0.00 & $0 ; 56$ \\
\hline Multi-method approaches & 5 & 0.80 & $0.58 ; 1.10$ & -1.39 & 39.66 & $0 ; 77$ \\
\hline
\end{tabular}




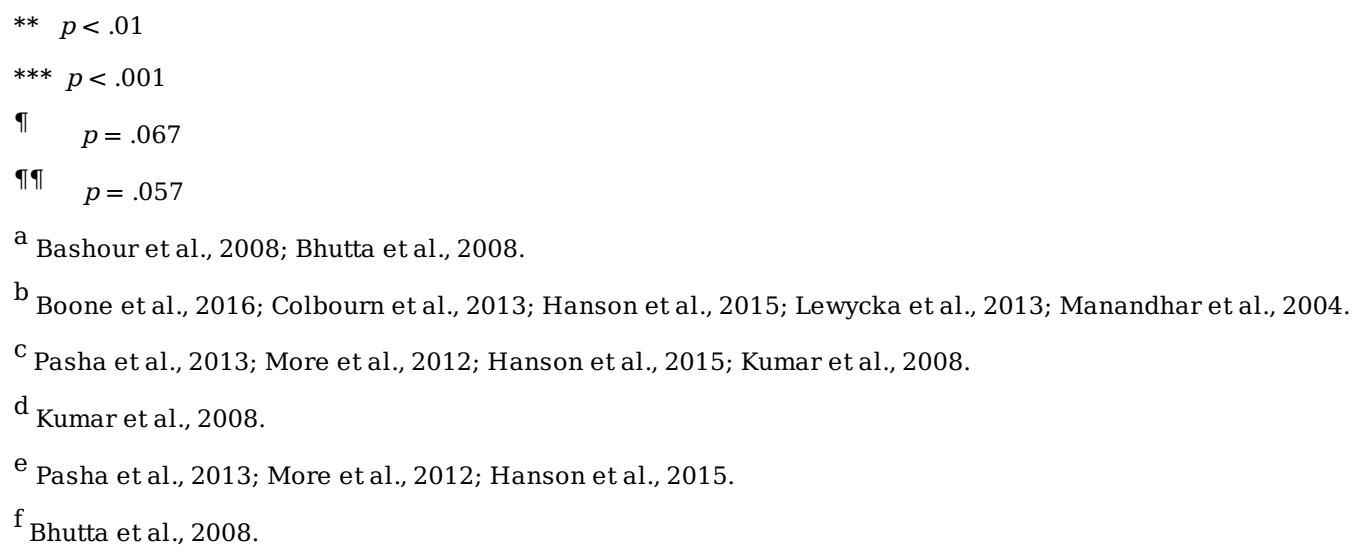

Sensitivity analyses

Sensitivity analyses were conducted by excluding those studies demonstrating a high risk of bias (see Table 3 for details). The overall effect changed only slightly to $14 \%$ while heterogeneity was slightly reduced to $77 \%(\mathrm{RR} 0.86,95 \% \mathrm{Cl} 0.78-0.95, p<$ $.01 ; 277,95 \% \mathrm{Cl} 64-84)$. Excluding all studies with high or medium risk of bias led to a heterogeneity level of $67 \%$ while the effect remained about the same (RR $0.84,95 \% \mathrm{Cl} 0.76-0.92, p<.001 ; 267,95 \% \mathrm{Cl} 29-81)$.

Investigation by intervention type indicated that comprehensive multi-method approaches led to the most pronounced risk reduction of NNM by about $18 \%$ compared to control groups (RR $0.82,95 \% \mathrm{Cl} 0.68-0.99, p<.05$ ). However, these studies were also marked by the highest heterogeneity $\left({ }^{2} 86,95 \% \mathrm{Cl} 70-92\right)$. Group-based intervention approaches led to a just nonsignificant reduction of $15 \%$ compared to controls (RR $0.85,95 \% \mathrm{Cl} 0.72-1.01, p<.01 ; P^{2} 84,95 \% \mathrm{Cl} 68-90$ ). The effect of individualised interventions (home-visits) was low and non-significant (RR 0.93, 95\% $\mathrm{Cl} 0.81-1.06, p<0.1)$.

Publication bias

The analysis of funnel plots did not point at significant risk of publication bias through unpublished studies with negative findings. 175 studies with null results would be needed in order for the overall effect on neonatal mortality to become insignificant (classic fail-safe $\mathrm{N}, p s<.001$ ). It is thus unlikely that publication bias posed a substantial threat to the validity of the results.

Maternal mortality

The forest plot of 14 studies reporting on maternal mortality is shown in Figure A-2. There was a significant effect (RR 0.79 , $95 \% \mathrm{Cl} 0.68-0.93, p<.01)$, suggesting an almost $21 \%$ lower risk of maternal death in intervention compared to control groups. Heterogeneity among this sample of studies was low $(27,95 \% \mathrm{Cl} 0-51)$.

Sensitivity analyses

Excluding the one study demonstrating high risk of bias (Bhutta et al., 2008) did not change the effect but led to slightly increased heterogeneity (RR $0.79,95 \% \mathrm{Cl} 0.66-0.94, p<.01 ; P^{2} 12.08,95 \% \mathrm{Cl} 0-55$ ).

Investigation of intervention type suggested that group-based approaches were most effective with the potential of reducing the likelihood of maternal death by almost $27 \%$ (RR $0.73,95 \% \mathrm{Cl} 0.58-0.92 ; p<.01)$. Multi-method approaches reduced the likelihood of death by about $20 \%$, however not significant (RR $0.80,95 \% \mathrm{Cl} 0.58-1.10, p>0.1$ ). Heterogeneity in both categories was very low and non-significant (Table 3). Only one study in the maternal mortality analysis used an individual approach (Tomlinson et al., 2014), therefore no sub-analysis was conducted.

Publication bias 
Inspection of funnel plots did not suggest risk of publication bias. 120 studies with null results would be needed for the overall effect on maternal mortality to become insignificant $(p s<.001)$. It is unlikely that publication bias posed a substantial threat to the validity of the results.

\section{Discussion}

The results of this systematic review and meta-analysis add to a series of analyses demonstrating the potential of psychosocial interventions to positively influence a range of outcomes related to sexual and reproductive health in low-and middle-income countries (Turner et al., in press; Riedel et al., subm. 2019), including birth-related mortality (neonatal and maternal).

Among the 21 included RCTs, the risk of neonatal death was almost 15\% lower in intervention compared to control groups. Based on the current global neonatal mortality rate of 18 deaths per 1,000 livebirths (UNICEF, 2018), a reduction of $15 \%$ corresponds to 2.7 prevented deaths per 1,000 livebirths and thus an approximated mortality rate of 15 . As a reference, a global rate of 15 has been set as the accelerated-action target for 2020 by the 'Every Newborn' WHO and UNICEF joint action plan (WHO, 2014; Lawn et al., 2014).

The risk of maternal mortality, reported in 14 studies, was reduced by almost $21 \%$ in the intervention compared to the control groups. This effect translates into 46 prevented maternal deaths per 100,000 live births in relation to the assumed global rate of 216 (UNICEF, 2017). This result fits in with the picture that the rate of improvements is faster with regard to maternal as compared to neonatal mortality (Bhutta et al., 2014).

Our estimate for neonatal mortality has to be taken with caution as it is accompanied by high heterogeneity, probably due to the variety of intervention types that have been included as 'psychosocial' in this analysis. In turn, among studies for maternal mortality, heterogeneity was low. Promising is the finding that, overall, most studies adhered to reasonable to good quality standards and that excluding studies with higher risk of bias increased the overall effect while reducing heterogeneity.

In the present sample of studies selected for this review, interventions with multi-method approaches showed the highest significant reduction of neonatal mortality, as demonstrated in our sensitivity analyses. These studies consisted of comprehensive intervention packages targeting the intervention clusters through a variety of channels. These entailed, for example: 1) establishing culturally contextualised group-based information and education events (e.g. Boone et al., 2017: folk song and dance used to promote child and maternal health themes; Boone et al., 2016: visualisation methods; Bhutta et al. 2011: video-docudrama on pregnancy and newborn care in the local language; Kumar et al., 2008: folk song group meetings);

2) involving community stakeholders through participatory action cycles to address birth-related problems (Boone et al., 2016; Kumar et al., 2008; Pasha et al., 2013); 3) targeting expectant mothers and families directly through home visitation and 4) involving CHWs and traditional birth attendants (Bhutta et al., 2008 \& 2011; Boone et al., 2016 \& 2017; Kumar et al., 2008; Pasha et al., 2013). The facilitation of participatory learning and action cycles (e.g., Tripathy et al., 2016) was also a major component of most studies categorised as 'group-based' in this review, the effect for which was not as pronounced. However, the lack of an effect among studies employing individual approaches - featuring home visits to expectant or new mothers as the main component - supports the interpretation that targeting the social orbit rather than the individual only could have better impact in reducing mortality rates. It must be considered that the strategies described above are examples of elements in comprehensive studies which also entailed other elements, so that the effective mechanisms of change remain difficult to pinpoint. Yet, one of the mechanisms that was common to the most effective interventions was bringing about a shift in perception and awareness of the relevance of birth-related care on a societal scale, which was shown to be necessary to ensure continued progress (Kumar et al., 2012).

There was little heterogeneity among studies reporting on maternal mortality, possibly because there was only one individual trial among them. Due to insufficient numbers of studies, it was not possible to investigate the influence of intervention type in much detail. The only feasible sub-analysis showed that group-based approaches - focused on facilitating participatory learning and action cycles through group meetings with women and other community members - were most effective in

Page $10 / 16$ 
reducing maternal mortality risk, a finding that is in line with previous research (Prost et al., 2013). As authors argued, the active engagement of other community members (and not just women) enhances social awareness and alertness which can lead to maternal health problems being addressed more appropriately (e.g. Manandhar et al., 2004; More et al., 2012; Tripathy et al., 2010). However, more trials are needed to test such assumptions of change mechanisms more thoroughly.

The following limitations of this meta-analytic review have to be considered. One is the existence of substantial heterogeneity among studies in the NNM-analysis, which has been discussed above. With more high-quality RCTs conducted, future analyses will allow to more closely examine sources of heterogeneity, for example by grouping studies more precisely based on regional economic and healthcare situations or on intervention type. The most effective type of intervention for NNM risk reduction were large-scale multi-method interventions, but with only six studies this analysis had also least power. It should be noted that multi-method interventions come at the price of increased risk of bias due to complex reporting systems and make it difficult to identify the main mechanism(s) of change. Although the multi-method studies included here did share similar elements, they are still arguably difficult to compare in their entirety. Finally, it must be noted that we were not always able to extract direct estimates of RR adjusted for clustering, because not all studies provided this information. It is possible that the pooled estimate would be different (although likely not substantially) if only such more precise estimates had been used. This has to also be taken into consideration when interpreting our results and planning implementation studies.

The results of this analysis demonstrated the effectiveness of socially inclusive strategies targeting the demand side of health care (communities, families, mothers) to reduce neonatal and maternal mortality rates in LMICs. Implementation efforts can draw from a solid base of effective approaches but should continue to be rigorously planned and documented to confirm the long-lasting emergent effects on targeted communities.

\section{Declarations}

\section{Ethics approval and consent to participate}

Not applicable.

\section{Consent for publication}

Not applicable.

\section{Availability of data and materials}

The datasets during and/or analysed during the current study available from the corresponding author on reasonable request.

\section{Competing interests}

The authors have declared that no competing interests exist. The authors alone are responsible for the views expressed in this article and they do not necessarily represent the views, decisions or policies of the funding bodies or institutions with which they are affiliated.

\section{Funding}

This work was funded by the Department of Reproductive Health and Research (RHR), including the UNDP/UNFPA/UNICEF/WHO/World/Bank Special programme of research, development and research training in human reproduction (HRP). The funders had no role in study design, data collection and analysis, decision to publish, or preparation of the manuscript.

\section{Authors' contributions}


DT, PC and EK developed the search string and performed the database searches. DT and ER conducted the study selection and data extraction. CM, ER and DT conducted the risk of bias assessment. ER performed the analyses and drafted the manuscript. All authors participated in the discussion of structure, selection criteria and quality assessment. All authors reviewed and approved of the manuscript.

\section{Acknowledgements}

To be added after review.

\section{References}

Azad, K., Barnett, S., Banerjee, B., Shaha, S., Khan, K., Rego, A. R., ... Costello, A. (2010). Effect of scaling up women's groups on birth outcomes in three rural districts in Bangladesh: a cluster-randomised controlled trial. Lancet (London, England), 375(9721), 1193-1202. https://doi.org/10.1016/S0140-6736(10)60142-0

Baqui, A. H., El-Arifeen, S., Darmstadt, G. L., Ahmed, S., Williams, E. K., Seraji, H. R., ... Black, R. E. (2008). Effect of communitybased newborn-care intervention package implemented through two service-delivery strategies in Sylhet district, Bangladesh: a cluster-randomised controlled trial. Lancet (London, England), 371(9628), 1936-1944. https://doi.org/10.1016/S01406736(08)60835-1

Bashour, H. N., Kharouf, M. H., AbdulSalam, A. A., El Asmar, K., Tabbaa, M. A., \& Cheikha, S. A. (2008). Effect of postnatal home visits on maternal/infant outcomes in Syria: A randomized controlled trial. Public Health Nursing, 25(2), 115-125. https://doi.org/10.1111/j.1525-1446.2008.00688.x

Bhutta, Z. A., Cousens, S., Adam, T., Walker, N., de Bernis, L., \& Team, L. N. S. S. (2005). Evidence-based, cost-effective interventions: how many newborn babies can we save?. Lancet, 365(9463), 977-988. Retrieved from http://ovidsp.ovid.com/ovidweb.cgi?T=JS\&PAGE=reference\&D=med5\&NEWS=N\&AN=15767001

Bhutta, Z. A., Das, J. K., Bahl, R., Lawn, J. E., Salam, R. A., Paul, V. K., ... Walker, N. (2014). Can available interventions end preventable deaths in mothers, newborn babies, and stillbirths, and at what cost? The Lancet, 384(9940), 347-370. https://doi.org/10.1016/S0140-6736(14)60792-3

Bhutta, Z. A., Memon, Z. A., Soofi, S., Salat, M. S., Cousens, S., \& Martines, J. (2008). Implementing community-based perinatal care: results from a pilot study in rural Pakistan. Bulletin of the World Health Organization, 86(6), 452-459.

Bhutta, Z. A., Soofi, S., Cousens, S., Mohammad, S., Memon, Z. A., Ali, I., ... Martines, J. (2011). Improvement of perinatal and newborn care in rural Pakistan through community-based strategies: A cluster-randomised effectiveness trial. The Lancet, 377(9763), 403-412. https://doi.org/10.1016/S0140-6736(10)62274-X

Boone, P., Eble, A., Elbourne, D., Frost, C., Jayanty, C., Lakshminarayana, R., ... Reddy, P. (2017). Community health promotion and medical provision for neonatal health-CHAMPION cluster randomised trial in Nagarkurnool district, Telangana (formerly Andhra Pradesh), India. PLoS Medicine, 14(7), e1002324. https://doi.org/10.1371/journal.pmed.1002324

Boone, P., Elbourne, D., Fazzio, I., Fernandes, S., Frost, C., Jayanty, C., ... Walker, P. R. (2016). Effects of community health interventions on under-5 mortality in rural Guinea-Bissau (EPICS): A cluster-randomised controlled trial. The Lancet Global Health, 4(5), e328-e335. https://doi.org/10.1016/S2214-109X(16)30048-1

Colbourn, T., Nambiar, B., Bondo, A., Makwenda, C., Tsetekani, E., Makonda-Ridley, A., ... Costello, A. (2013). Effects of quality improvement in health facilities and community mobilization through women's groups on maternal, neonatal and perinatal mortality in three districts of Malawi: MaiKhanda, a cluster randomized controlled effectiveness trial., 5(3), 180-195. https://doi.org/10.1093/inthealth/iht011

Page $12 / 16$ 
Darmstadt, G. L., Choi, Y., Arifeen, S. E., Bari, S., Rahman, S. M., Mannan, I., ... Baqui, A. H. (2010). Evaluation of a clusterrandomized controlled trial of a package of community-based maternal and newborn interventions in Mirzapur, Bangladesh. PloS One, 5(3), e9696-e9696. https://doi.org/10.1371/journal.pone.0009696

Fottrell, E., Azad, K., Kuddus, A., Younes, L., Shaha, S., Nahar, T., ... Houweling, T. A. J. (2013). The effect of increased coverage of participatory women's groups on neonatal mortality in Bangladesh: A cluster randomized trial. JAMA Pediatrics, 167(9), 816-825. https://doi.org/10.1001/jamapediatrics.2013.2534

Hanson, C., Manzi, F., Mkumbo, E., Shirima, K., Penfold, S., Hill, Z., ... Schellenberg, J. (2015). Effectiveness of a Home-Based Counselling Strategy on Neonatal Care and Survival: A Cluster-Randomised Trial in Six Districts of Rural Southern Tanzania. PLoS Medicine, 12(9), e1001881-e1001881. https://doi.org/10.1371/journal.pmed.1001881

Higgins, J.P.T. \& Green, S. (2011). Cochrane Handbook for Systematic Reviews of Interventions, Version 5.1.0 [updated March 2011]. The Cochrane Collaboration. Available from www.handbook.cochrane.org.

Houweling, T. A. J., Azad, K., Younes, L., Kuddus, A., Shaha, S., Haq, B., ... Costello, A. (2011). The effect of participatory women's groups on birth outcomes in Bangladesh: Does coverage matter? Study protocol for a randomized controlled trial. Trials, 12(1), 208. https://doi.org/10.1186/1745-6215-12-208

Jackson D, Turner R. Power analysis for random-effects meta-analysis. Res Synth Methods. 2017;8(3):290-302.

Kirkwood, B. R., Manu, A., Tawiah-Agyemang, C., ten Asbroek, G., Gyan, T., Weobong, B., ... Hill, Z. (2010). NEWHINTS cluster randomised trial to evaluate the impact on neonatal mortality in rural Ghana of routine home visits to provide a package of essential newborn care interventions in the third trimester of pregnancy and the first week of life: Trial protoco. Trials, 11(May). https://doi.org/10.1186/1745-6215-11-58

Kirkwood, B. R., Manu, A., ten Asbroek, A. H. A., Soremekun, S., Weobong, B., Gyan, T., ... Hill, Z. (2013). Effect of the Newhints home-visits intervention on neonatal mortality rate and care practices in Ghana: a cluster randomised controlled trial. Lancet (London, England), 381(9884), 2184-2192. https://doi.org/10.1016/S0140-6736(13)60095-1

Kirkwood, B., \& Bahl, R. (2013). Can women's groups reduce maternal and newborn deaths? The Lancet, 381(9879), $12-14$. https://doi.org/10.1016/S0140-6736(13)60985-X

Kumar, V., Kumar, A., Das, V., Srivastava, N. M., Baqui, A. H., Santosham, M., \& Darmstadt, G. L. (2012). Community-driven impact of a newborn-focused behavioral intervention on maternal health in Shivgarh, India. International Journal of Gynecology and Obstetrics, 117(1), 48-55. https://doi.org/10.1016/j.ijgo.2011.10.031

Kumar, V., Mohanty, S., Kumar, A., Misra, R. P., Santosham, M., Awasthi, S., ... Darmstadt, G. L. (2008). Effect of communitybased behaviour change management on neonatal mortality in Shivgarh, Uttar Pradesh, India: a cluster-randomised controlled trial. Lancet (London, England), 372(9644), 1151-1162. https://doi.org/10.1016/S0140-6736(08)61483-X

Lawn, J. E., Blencowe, H., Oza, S., You, D., Lee, A. C. C., Waiswa, P., ... Cousens, S. N. (2014). Every newborn: Progress, priorities, and potential beyond survival. The Lancet, 384(9938), 189-205. https://doi.org/10.1016/S0140-6736(14)60496-7

Lewycka, S., Mwansambo, C., Rosato, M., Kazembe, P., Phiri, T., Mganga, A., ... Costello, A. (2013). Effect of women's groups and volunteer peer counselling on rates of mortality, morbidity, and health behaviours in mothers and children in rural Malawi (MaiMwana): A factorial, cluster-randomised controlled trial. The Lancet, 381(9879), 1721-1735. https://doi.org/10.1016/S0140-6736(12)61959-X

Manandhar, D. S., Osrin, D., Shrestha, B. P., Mesko, N., Morrison, J., Tumbahangphe, K. M., ... Costello, A. M. de L. (2004). Effect of a participatory intervention with women's groups on birth outcomes in Nepal: cluster-randomised controlled trial. Lancet (London, England), 364(9438), 970-979. https://doi.org/10.1016/S0140-6736(04)17021-9

Page $13 / 16$ 
More, N. S., Bapat, U., Das, S., Alcock, G., Patil, S., Porel, M., ... Osrin, D. (2012). Community mobilization in Mumbai slums to improve perinatal care and outcomes: a cluster randomized controlled trial. PLoS Medicine, 9(7), e1001257-e1001257. https://doi.org/10.1371/journal.pmed.1001257

Nair, N., Tripathy, P., Sachdev, H. S., Pradhan, H., Bhattacharyya, S., Gope, R., ... Prost, A. (2017). Effect of participatory women's groups and counselling through home visits on children's linear growth in rural eastern India (CARING trial): a clusterrandomised controlled trial. The Lancet Global Health, 5(10), e1004-e1016. https://doi.org/10.1016/S2214-109X(17)30339-X

Riedel, E. M. C., Turner, D. T., Kobeissi-Hassan, L., Karyotaki, E., Harper-Shehade, M., Garcia-Moreno, C., Say, L. \& Cuijpers, P. (submitted). The impact of psychosocial interventions on condom/contraceptive use and sexual behaviour in LMICs: Systematic review and meta-analysis.

Pasha, O., McClure, E. M., Wright, L. L., Saleem, S., Goudar, S. S., Chomba, E., ... Goldenberg, R. L. (2013). A combined community- and facility-based approach to improve pregnancy outcomes in low-resource settings: a Global Network cluster randomized trial. BMC Medicine, 11, 215. https://doi.org/10.1186/1741-7015-11-215

Persson, L. Å., Nga, N. T., Målqvist, M., Thi Phuong Hoa, D., Eriksson, L., Wallin, L., ... Ewald, U. (2013). Effect of Facilitation of Local Maternal-and-Newborn Stakeholder Groups on Neonatal Mortality: Cluster-Randomized Controlled Trial. PLoS Medicine, 10(5), 1-11. https://doi.org/10.1371/journal.pmed.1001445

Prost, A., Colbourn, T., Seward, N., Azad, K., Coomarasamy, A., Copas, A., ... Costello, A. (2013). Women's groups practising participatory learning and action to improve maternal and newborn health in low-resource settings: A systematic review and meta-analysis. The Lancet, 381(9879), 1736-1746. https://doi.org/10.1016/S0140-6736(13)60685-6

The World Bank (2018). World Bank Country and Lending Groups. Retrieved from https://datahelpdesk.worldbank.org/knowledgebase/articles/906519-world-bank-country-and-lending-groups [November 18].

Tomlinson, M., Doherty, T., ljumba, P., Jackson, D., Lawn, J., Persson, L. Å., ... Chopra, M. (2014). Goodstart: A cluster randomised effectiveness trial of an integrated, community-based package for maternal and newborn care, with prevention of mother-to-child transmission of HIV in a South African township. Tropical Medicine and International Health, 19(3), $256-266$. https://doi.org/10.1111/tmi.12257

Tripathy, P., Nair, N., Barnett, S., Mahapatra, R., Borghi, J., Rath, S., ... Costello, A. (2010). Effect of a participatory intervention with women's groups on birth outcomes and maternal depression in Jharkhand and Orissa, India: a cluster-randomised controlled trial. The Lancet, 375(9721), 1182-1192. https://doi.org/10.1016/S0140-6736(09)62042-0

Tripathy, P., Nair, N., Sinha, R., Rath, S., Gope, R. K., Rath, S., ... Prost, A. (2016). Effect of participatory women's groups facilitated by Accredited Social Health Activists on birth outcomes in rural eastern India: a cluster-randomised controlled trial. The Lancet Global Health, 4(2), e119-28. https://doi.org/10.1016/S2214-109X(15)00287-9

Turner, D. T., Riedel, E. M. C., Karyotaki, E., Cuijpers, P., Kobeissi-Hassan, L., \& Say, L. (in press). The impact of psychosocial interventions on intimate partner violence in LMICs. Systematic review and meta-analysis. Journal of Global Health.

UNICEF (2018). Neonatal Mortality. Retrieved from https://data.unicef.org/topic/child-survival/neonatal-mortality/ [November 2018]

UNICEF (2017). Maternal Mortality. Retrieved from https://data.unicef.org/topic/maternal-health/maternal-mortality/ [November 2018]

United Nations Organization (2018a). Sustainable Development Goals. Goal 3: Good Health and Well-Being. Retrieved from https://www.un.org/sustainabledevelopment/health/ [November 2018]. 
United Nations Organization (2018b). Every Woman Every Child. UN Commission on Life-Saving Commodities for Women and Children [website]. United Nations Foundation; 2012. http://everywomaneverychild.org/resources/un-commission-on-lifesaving-commodities [December 2018]

United Nations Organization (2016). The 2030 agenda for Sustainable Development. Retrieved from https://sustainabledevelopment.un.org/post2015/transformingourworld/publication [December 2018]

Villar, J., Farnot, U., Barros, F., Victora, C., Langer, A., \& Belizan, J. (1992). A randomized trial of psychosocial support during high-risk pregnancies. The New England Journal of Medicine, 327(18), 1266-1271.

World Health Organization (2017). Sexual health and its linkages to reproductive health: an operational approach. World Health Organisation. Retrieved from http://apps.who.int/iris/bitstream/10665/258738/1/9789241512886-eng.pdf?ua=1 [November 2018].

World Health Organization (2015). Global Strategy for Women's, Children's and Adolescents Health 2016-2030. Retrieved from https://www.who.int/life-course/publications/global-strategy-2016-2030/en/ [December 2018].

World Health Organization, \& UNICEF (2009). WHO/UNICEF Joint statement: Home visits for the newborn child: a strategy to improve survival. World Health Organization, 1-8.

World Health Organization (2014). Every Newborn Action Plan. World Health Organization Library Cataloguing-in-Publication Data, 58.

\section{Figures}


Records identified through database searching: $\mathbf{8 , 2 8 5}$

PubMed: 1,515 Psyclnfo: 907

Embase: 2,833 CINAHL: 1,588

POPLINE: 43 Global Health: 1,277 Additional records identified through

Global Index Medicus: 122

other sources: $\mathbf{9}$

Records after duplicates removed: 6,450

Excluded after screening titles and abstracts:

5,389

Publications retrieved: $\mathbf{1 , 0 6 1}$

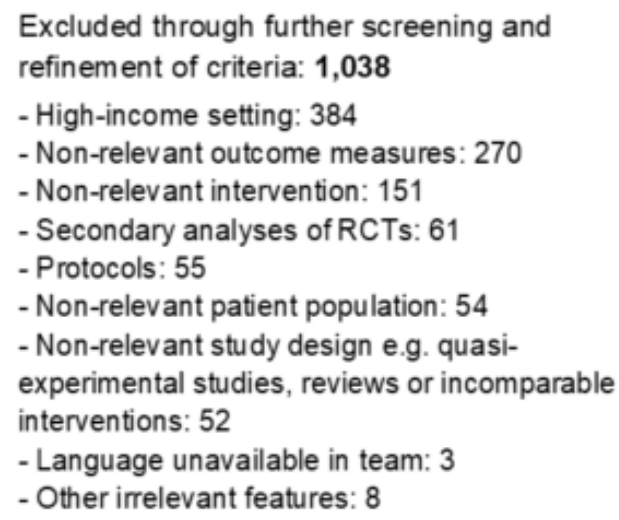

Studies included for data extraction: 23

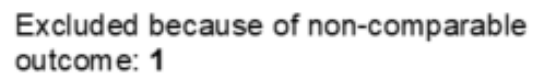

RCTs included in quantitative syntheses: 22

- Neonatal mortality: 21

- Maternal mortality: 14

\section{Figure 1}

Flow chart of the study selection process, following PRISMA-guidelines (Preferred Reporting Items for Systematic Reviews and Meta-Analyses).

\section{Supplementary Files}

This is a list of supplementary files associated with this preprint. Click to download.

- FigureA2.docx

- FigureA1.docx

- AppendixA3exemplarysearchstring.docx 